\title{
AUTOEFICACIA Y ACTIVIDAD FÍSICA EN NIÑOS MEXICANOS CON OBESIDAD Y SOBREPESO
}

\section{SELF-EFFICACY AND PHYSICAL ACTIVITY IN MEXICAN CHILDREN WITH OBESITY AND OVERWEIGHT}

\author{
Jauregui Ulloa, E.E. ${ }^{1}$, López Taylor, J.R. ${ }^{1}$, Mací as Serrano, A.N. ${ }^{1}$, Porras Rangel, S. ${ }^{1}$, \\ Reynaga-Estrada, P.2, Morales Acosta, J.J.2 , Cabrera González, J.L. ${ }^{3}$ \\ edtna01@yahoo.com.mx
}

\begin{abstract}
${ }^{1}$ Instituto de Ciencias Aplicadas a la Actividad Fí sica y al Deporte. Departamento de Ciencias del Movimiento Humano. Universidad de Guadalajara, Jalisco, México. ${ }^{2}$ Laboratorio de Psicologí a de la Actividad Fí sica y Deporte. Departamento de Ciencias del Movimiento Humano. Universidad de Guadalajara, Jalisco, México. ${ }^{3}$ Departamento de Reproducción Humana, Crecimiento y Desarrollo Infantil. Universidad de Guadalajara, Jalisco, México.
\end{abstract} Recibido: Octubre/2013 - Aceptado: Noviembre/2013.

\section{Resumen}

El estudio es una investigación de corte exploratorio, descriptivo como experimento de campo pre y post intervención en grupos pareados para trabajar con niños que tienen obesidad y sobrepeso y sus familias (el grupo de intervención) versus la ausencia de sus familias (grupo control), para evaluar los niveles de autoeficacia en un programa universitario de actividad física. Se encontraron diferencias significativas entre ambos grupos en la pre y la post intervención. Los escasos trabajos de intervención encontrados similares a este estudio en niños con obesidad y sus familias permite comprender las dificultades prácticas reportadas en este y otros estudios tienen que ver con los instrumentos aplicados y la metodología empleada y nos llevan a la conclusión de considerar en futuros estudios variables como autoestima, auto imagen y apoyo social de los niños con obesidad y sus familias.

Palabras clave:Obesidad infantil, autoeficacia, actividad física.

\begin{abstract}
This is an exploratory, descriptive, research, designed as a field test before and after intervention in non-coupled groups, to work with overweight and obese children and their families (intervention group); versus working without the individual's family members, to assess the degree of self-efficacy for the physical activity Program. There were significative differences of self efficacy changes among two groups before and after intervention. Some difficulties related to methodology and instruments were found and practical difficulties were reported such a previous studies with similar interventions studies. Conclusions: Promoting physical requires considering to include in the future another intraindividual characteristics of the children and families such as self-steem, body image and social support environmental.
\end{abstract}

Keywords: children obesity, self-efficacy, physical activity.

${ }^{1}$ Este trabajo de investigación fue aprobado por el Comité de Ética, Investigación y Bioseguridad del Centro Universitario de Ciencias de la Salud de la Universidad de Guadalajara, bajo dictamen y número de registro CI-12609. Se obtuvo financiamiento gracias al apoyo otorgado por el Programa de Mejoramiento del Profesorado (PROMEP), con número 103.5/11/6834, en el marco de "Apoyo al fortalecimiento de Cuerpos Académicos en Formación" para el Cuerpo Académico de Actividad Física y Salud (UDG-CA-59). Los autores declaran que no existe conflicto de interés.

\section{INTRODUCCIÓN}

La obesidad se considera como uno de los principales problemas de salud mundiales de creciente prevalencia y gravedad. De acuerdo con la Organización Mundial de la Salud la frecuencia de la obesidad en el mundo se ha incrementado de forma importante en los últimos años. Según los reportes de la OCDE (por sus siglas en español: Organización para la Cooperación y Desarrollo Economico, 2013), a nivel mundial la población de México mayor de 20 años ocupa el segundo lugar con problemas de sobrepeso y obesidad; y la población infantil ocupa el primer lugar con sobrepeso. De 1980 a la fecha esta prevalencia se ha triplicado particularmente en la población adulta. El sobrepeso y la obesidad en niños menores de cinco años es del $9.7 \%$; en los niños de 5 a 11 años es de 34.6\%; y en jóvenes de 12 a 19 años es del 35\% (Gutierrez, Rivera, Shama, \& Villapando, 2012).

La obesidad es el resultado de un desequilibrio entre el aporte calórico de la ingesta y el gasto energético del metabolismo y la actividad física. Este aporte calórico es común que en los niños sea ricos en grasas saturadas, azúcar y sal (Olivares, 2006; Bresh, 2006); lo que conlleva una condición que implica exceso de grasa o tejido adiposo en el cuerpo y puede ocasionar serios problemas (Bravo y Cols., 2011).

La obesidad está asociada a la morbilidad y a mayor riesgo de mortalidad. Según Alvarado (2005) las consecuencias de la obesidad pueden ser físicas o psicológicas. En las consecuencias físicas es el factor más significativo para aumentar el riesgo de desarrollar enfermedades crónicas como los trastornos cardiovasculares, dislipidemia, hipertensión arterial y diabetes (Alvarado, 2005; Bresh, 2006; Bravo, Espinoza, Mancilla \& Tello, 2011; Olivares, 2006).

Dentro de las consecuencias psicológicas de la obesidad se muestra la relación con un alta prevalencia de comorbilidad psiquiátrica y se enlistan importantes alteraciones emocionales como miedo, inseguridad personal, pérdida de autoestima, desórdenes alimenticios, conductas adictivas, distorsión de la imagen corporal, frigidez e impotencia, perturbación emocional por hábitos de ingesta erróneos, así como tristeza, infelicidad, depresión, y elevados niveles de ansiedad (Alvarado, 2005; Bresh, 2006; Bravo, Espinoza, Mancilla \& Tello, 2011; Olivares, 2006; Seijas \& Feuchtman, 1997; Villaseñor, 2006). 
La obesidad es uno de los pocos trastornos que son visibles a los demás, y que constituye uno de los principales estigmas sociales, disminuyendo las oportunidades de empleo y educación, con el consiguiente costo psicológico individual; además se tiende a ridiculizar y castigar a estas personas. En particular los pacientes de nivel socioeconómico medioalto experimentan repetidos fracasos para controlar su peso. Luego de una pérdida de peso, vuelven a subirlo, lo que a veces se ve exacerbado por los mismos profesionales, que sugieren que dichas personas no tienen muchas ganas de bajar de peso, pues de lo contrario habrían hecho más esfuerzo. La carga emocional puede no desembocar en una depresión clínica, pero reduce la autoestima del paciente en algunas áreas de funcionamiento y afecta la calidad de vida general del individuo. La frustración y la vergüenza se intensifican en estos pacientes. (Seijas \& Feuchtman, 1997; Villaseñor, 2006).

\section{AUTOEFICACIA}

La autoeficacia se define como los juicios de cada individuo sobre sus capacidades, en base a los cuales organizará y ejecutará sus actos de modo que le permitan alcanzar el rendimiento deseado. La autoeficacia ha sido ampliamente discutida en la literatura de la psicología social para explicar la teoría de la motivación y el aprendizaje. Este concepto ha sido utilizado en la psicología, la enfermería y la medicina, como un importante predictor de conductas saludables para recuperarse de los problemas de salud o para evitar sus riesgos potenciales; $y$ en el funcionamiento de distintos campos como el realizar ejercicio o deporte, bajar de peso, el manejo de enfermedades crónicas, el uso de drogas, la actividad sexual, el fumar, la elección de carrera y el logro de metas académicas y del mundo laboral (Carrasco, 2002). De hecho, la autoeficacia demuestra ser un factor a de gran importancia frente a la motivación para adoptar conductas que promuevan la salud o el detener conductas nocivas para ésta (Bandura, 1997). Las investigaciones revelan que los altos niveles de autoeficacia tienen consecuencias beneficiosas para el funcionamiento del individuo y su bienestar general.

Los programas dirigidos a promover comportamientos saludables en la familia deben empezar en la infancia porque es el periodo evolutivo ideal para adoptar estilos de vida sanos (Aedo, 2009). A decir de Diaz y Cols. (2010), tratar la obesidad con programas intensivos en el estilo de vida durante la niñez tienen éxito relativo; porque, de acuerdo a Reyna (2012), las prácticas de alimentación y actividad física en esta edad repercuten en el estado de salud del nińo $y$, posteriormente, en la salud del adulto y en la economía del país.

La obesidad infantil y, si persiste en la edad adulta, su asociación con los factores de riesgo de las enfermedades crónicas y con el pobre éxito de su tratamiento, actualmente lleva a considerar a la autoeficacia como un elemento definitivo para comprender la relación de los cambios de conducta y los factores de riesgo de la enfermedades crónicas, que en los niños obesos pueden identificarse y atenderse si la personas percibe su propia capacidad para realizar las acciones recomendadas y lograr la conducta deseada (Olivares, 2006).

Distintos hallazgos indican que la autoeficacia funciona como mediadora entre las intenciones iniciales de realizar ejercicio y la actividad física realizada posteriormente. Aquellas personas con mayores niveles de autoeficacia se comprometen con mayor frecuencia en un programa regular de actividad física que aquellos con más bajos niveles de autoeficacia (Aedo, 2009). Los individuos con alta autoeficacia tienen sentimientos mucho más fuertes de efectividad para realizar el ejercicio y para comprometerse en ese momento en realizar una mayor cantidad de actividad física que los individuos con baja autoeficacia. Los individuos con alta autoeficacia percibida tienen más probabilidad de iniciar cuidados preventivos, buscan tratamientos tempranos y son más optimistas sobre la eficacia de estos (Grembowsky et al, 1993). En relación con el ejercicio se reporta que la autoeficacia es un mediador muy ligado en la realización de actividad física en niños y adolescentes (Dishman, Molt, RW, Saunders, \& col, 2004). Un programa de intervención que genere actitudes, respuestas y afectos positivos al ejercicio, servirá para que los deportistas mantengan altos niveles de motivación y el interés por la práctica del deporte aun cuando no sean atletas de elite, permitiendo al individuo mantener el deseo y el interés de continuar ejercitándose (Kwan, 2010).

Los criterios de éxito terapéutico deben contemplar no solo la reducción de peso como producto final, sino como un proceso continuo con resultados positivos en la calidad de vida, integrando activamente el ejercicio, una mayor aceptación de su imagen corporal y un cambio de hábitos de alimentación (Villaseñor, 2006).

Un tratamiento integral del paciente con sobrepeso $\mathrm{u}$ obesidad debe ser facilitarle las herramientas que le hagan posible un cambio de hábitos alimentarios y de estilo de vida, y también apoyarle con los elementos básicos para que logre un fortalecimiento interno que le ayude a obtener resultados óptimos y le impidan que una vez reducido el peso no vuelva a aumentar y le otorguen la seguridad y confianza necesaria para enfrentar posibles recaídas (Seijas \& Feuchtman, 1997).

El objetivo planteado para este estudio se estableció en los términos siguientes:

1) Evaluar la percepción de la autoeficacia en niños mexicanos con obesidad, antes y después de de participar en un programa universitario con apoyo y sin apoyo de la familia.

La pregunta inicial en este estudio se formuló de la 
siguiente manera:

1. ¿Qué diferencias en la percepción de autoeficacia presentan niños con obesidad antes y después de participar en un programa universitario de actividad física con y sin apoyo de la familia?

La hipótesis nula fue:

1. No existe diferencia en la percepción de autoeficacia en niños mexicanos con sobrepeso y obesidad antes y después de participar en un programa de actividad física con y sin apoyo de la familia.

\section{La hipótesis alternativa se planteó}

2. La diferencia en la percepción de autoeficacia en niños mexicanos con sobrepeso y obesidad es mayor en las evaluaciones finales que en las evaluaciones iniciales tras participar en un programa de actividad física con y sin apoyo de la familia.

\section{MÉTODO}

Estudio exploratorio cuasi experimental o experimento de campo, longitudinal, con modelo de pre- post intervención, en dos grupos pareados: un grupo de intervención (con apoyo de la familia) y un grupo control (sin apoyo de la familia). El primer grupo constó de 46 niños de los cuales 33 eran del sexo femenino y 13 masculino. Después de la intervención, solo pudo considerarse a 11 individuos debido a que 21 no realizaron las dos evaluaciones y 14 de ellos no tuvieron permanencia ni continuidad en el programa, a pesar de haber realizado las dos evaluaciones. El segundo grupo constó de 20 niños, de los cuales solo permanecieron 9 sujetos: 3 de sexo femenino y 6 masculino. Los dos grupos con antecedentes familiares de obesidad. Se inició con 66 niños con sobrepeso y obesidad con edades entre 6 a 15 años, pero la evaluación se concluyó con 20 niños en los dos grupos.

El grupo control fue seleccionado previa convocatoria por medio de correos, carteles y llamadas telefónicas del programa por los cuales acudieron al Instituto de Ciencias Aplicadas a la Actividad Física y del Deporte de la Universidad de Guadalajara, donde se les explicó la finalidad del proyecto y se les pidió firmar la carta de consentimiento para participar en el estudio. De forma aleatorizada se definieron los casos del listado de familias (de intervención con asistencia de la familia) y controles (sin asistencia de la familia) en pares de listado de número aleatorios en bloques de dos.

El Programa universitario de actividad física tuvo 24 semanas de duración; quienes asistían dos veces por semana de 2 horas por sesión y recibían sesiones de activación, basquetbol, volibol, futbol y recreación. De esas 4 horas, una hora a la semana era de natación. La variable que diferenció a los grupos fue la inclusión de los miembros de la familia (intervención) o su ausencia (control). Las estrategias del programa fueron dirigidas a promover experiencias de aprendizaje haciéndolos vivir experiencias exitosas en su desempeño físico, persuasión directa con sesiones fuertes promoviendo sus habilidades y capacidades físicas y exposición con otros niños con sobrepeso y obesidad. Se evaluó la asistencia de los padres como indicador de la influencia social, determinándose ésta por una lista de asistencia y registrándose la presencia, de padre, madre, u otro familiar durante todo el programa.

Las mediciones antropométricas fueron basadas en la metodología propuesta por International Society for Advancement of Kinanthropometry (ISAK) (Norton \& Olds, 1996). Todos los evaluadores fueron estandarizados en la técnica, con un error técnico de medición menor para peso y estatura. La estatura fue medida usando un estadiómetro marca SECA (gmbh \& Co kg, Hamburgo Alemania), registrándose lo más cercano a $0.1 \mathrm{~cm}$. El peso corporal fue medido con una báscula marca Tanita HD-313 (Tokio, Japón), la cual se colocó sobre una base de madera para asegurar la regularidad de la superficie. La precisión en la medición del peso fue de $0.1 \mathrm{~kg}$. El Índice de Masa Corporal (IMC) fue calculado con el peso en kilogramos (kg) / estatura (m2). Se clasificó el peso corporal de acuerdo a lo resultante del IMC acorde a la clasificación internacional en sobrepeso si los niños tuvieron un IMC mayor de la percentil $>85$ y con obesidad cuando el IMC fue mayor al percentil > 95 para los puntos de corte para la edad y sexo (Cole, Bellizi, Flegal, \& Dietz, 2000).

\section{INSTRUMENTO}

Se utilizó el cuestionario de autoeficacia para la actividad física (Saunders y cols., 1997). Este instrumento consta de 17 ítems que el sujeto debe responder con una escala Likert de 5 opciones (muy en desacuerdo, desacuerdo, ni acuerdo, ni desacuerdo, de acuerdo, muy de acuerdo). La escala se califica dando una puntuación de 1 a 5 en cada ítem dependiendo de la respuesta, de esta manera Muy en desacuerdo equivaldría a 1 , Desacuerdo a 2 y así sucesivamente. La suma total de los ítems proporciona el resultado final del cuestionario. La consistencia interna de confiabilidad para los componentes de búsqueda de apoyo, barreras y alternativas positivas fueron de 0.71 $(\mathrm{n}=319), 0.71(\mathrm{n}=323)$ y $0.54(\mathrm{n}=321)$ respectivamente. Los datos de validación alfa fueron 0.52 , 0.55 y 0.62 respectivamente. La prueba de confiabilidad del testretest las escalas fueron entre del 0.76, 0.82 y 0.61 respectivamente. Para la búsqueda de apoyo y barreras existió débil correlación pero significativa en un 0.25 ; La búsqueda de apoyo y alternativas positivas correlacionaron significativamente en un 0.41 ; las barreras y alternativas positivas correlacionaron significamente en un 0.38 (Saunders y cols., 1997).

\section{PROCEDIMIENTO}

Se aplicó una entrevista al inicio del estudio así como el conjunto de pruebas anteriormente mencionadas y al final del estudio se volvió a aplicar el 
conjunto de pruebas para medir autoeficacia y la autoestima con la finalidad de comparar resultados iniciales y si los talleres aplicados a los participantes predijeron cambios en ellos.

En el grupo control, debido a las deserciones por el tipo de estudio cuasi experimental como experimento de campo existió dificultad de controlar la variable de asistencia y permanencia, en el cuestionario de Autoeficacia para la actividad física, de Saunders y col., (1997), pocos nińos respondieron.

Una vez aplicadas las pruebas, se capturaron los datos, y posteriormente se realizó el análisis estadístico. Con base al percentil 25, 50 y 75 se definieron los niveles bajo, medio y alto.

Dentro del procedimiento, podemos establecer las fases o etapas del proyecto, las cuales fueron:

1. Búsqueda de información documental

2. Realización de Fichas bibliográficas

3. Intervención profesional directa con población y trabajo de campo

\section{Aplicación de encuestas y mediciones}

5. Captura de datos

6. Análisis y procesamiento de datos y tabulación de resultados:

7. Redacción, discusión y conclusiones

\section{RESULTADOS}

Se aplicó el instrumento de autoeficacia de Saunders, al que se consideró grupo control con nueve niños con edades de los ocho a los doce años $(\mathrm{M}=9.6 ; \mathrm{DE}=1.5)$; en su mayoría hombres $(6=66 \%)$ y menor porcentaje de mujeres $(3=33.3 \%)$. Mientras que el grupo de intervención contó con 11 nińos de nueve a 16 años $(\mathrm{M}=9.25 ; \mathrm{DE}=4.65)$, con menor presencia de hombres (3, que representa el $27.27 \%$ ) que de mujeres (8, que representa el $72.72 \%)$.

En la tabla 1, se pueden observar los valores de los niveles, de acuerdo al grupo de respuestas de los grupos control y de intervención, tanto en la evaluación inicial como la final. Llama la atención el ligero aumento en las puntuaciones de la media para los niveles en la percepción de autoeficacia en ambos grupos y el descenso en la desviación estándar (DE).

Tabla 1. Nivel de autoeficacia, en el grupo control ( $n=9)$ y en el grupo de intervención ( $n=11)$, antes y después de participar en el Programa Universitario de Actividad Física.

\begin{tabular}{clllllll}
$\begin{array}{c}\text { Grupo de } \\
\text { estudio }\end{array}$ & $\begin{array}{c}\text { Etapa de } \\
\text { Evaluación }\end{array}$ & $\boldsymbol{M}$ & \multicolumn{1}{c}{ DE } & \multicolumn{1}{c}{ Bajo } & $\begin{array}{c}\text { Nivel } \\
\text { medio }\end{array}$ & Alto \\
\hline Control & Inicial & 68.67 & 10.332 & $<60.50$ & $61-79$ & $\geq 80$ \\
& Final & 70.11 & 8.609 & $<62.50$ & $63-77$ & $\geq 78$ \\
\multirow{4}{*}{ Intervención } & Inicial & 62.18 & 16.104 & $<62$ & $63-79$ & $\geq 80.75$ \\
& Final & 73 & 10.50 & $<58.50$ & $59-78$ & $\geq 79.75$ \\
\hline
\end{tabular}

\section{Análisis estadístico}

Se realizó el análisis de comparación de medias en la prueba de autoeficacia de Saunders, utilizando la prueba de hipótesis para medias con datos emparejados, los cuales se representan con las evaluaciones iniciales y finales para los sujetos. De esta forma los datos o variables son dependientes o pareadas porque se les aplica la evaluación a los mismos sujetos antes y después de la intervención. Las hipótesis son:

Ho: $\mu \mathrm{d}=0 \quad$ (Hipótesis nula)

Ha: $\mu \mathrm{d}>0 \quad$ (Hipótesis alternativa)

$\mu \mathrm{d}$ es la media o promedio de las diferencias pareadas.

El estadístico de prueba que se utiliza es:

$\mathrm{t}=\frac{\sqrt{n}}{s_{d}}\left(D-\mu_{d 0}\right)$ (Quevedo y Pérez, 2008) t es el estadístico de prueba para la distribución $t$ de Student para medias pareadas o emparejadas.

$\mathrm{D}$ es el promedio o la media de las diferencias entre los pares.

$\mathrm{N}$ es el número de parejas, en este caso $\mathrm{n}=11$.

sd es la desviación estándar de las diferencias pareadas.

Con un nivel de significancia del 5\%, se observó que la intervención si mejoró los puntajes de autoeficacia de Saunders, porque la evaluación posterior (Variable 1) fue significativamente mayor que la evaluación diagnóstica (variable 2). Ver Tabla 2. 
Coeficiente de correlación de Pearson

Diferencia hipotética de las medias

Estadístico t

\section{DISCUSIÓN Y CONCLUSIONES}

En virtud de los objetivos trazados, éstos se cumplieron en función de la descripción de los niveles de autoeficacia, a partir de las respuestas de los niños participantes en los grupos control y de intervención en las fases de evaluación inicial (pre intervención) y final (post intervención). Se observaron diferencias significativas entre los valores de la prueba de autoeficacia de Saunders pre y post intervención, de esta forma se concluye que la intervención produjo resultados favorables porque los valores de la prueba aumentaron de 62.18 a 73. No obstante, es importante destacar el gran porcentaje de no respuesta que existió, debido a la dificultad de comprensión de los niños respecto a las escalas e instrumentos utilizados, lo que puede llevarnos a pensar en un posible sesgo de aplicación. También es de señalar la dificultad de mantener la permanencia y constancia en el programa de intervención a los niños y sus familiares. Sin embargo, gracias a este estudio de carácter exploratorio y descriptivo se puede rescatar la experiencia para implementar estrategias e instrumentos que permitan resolver las dificultades vividas en el estudio.

En este estudio fue evidente el abandono y deserción de los nińos del grupo control, en este caso fue del $55 \%$, mientras que en el grupo de intervención fue del $76.08 \%$. Es importante hacer un análisis más profundo acerca de las causas del abandono y deserción al programa para establecer estrategias que mejoren la adherencia a la propuesta de intervención.

Por otra parte, coincidimos con Kwan (2010), en que los resultados sugieren que una respuesta afectiva positiva al ejercicio esta prospectivamente asociada con una mayor motivación a ejercitarse, porque aquellos que respondieron a una serie más aguda de ejercicio son aquellos que experimentaron una mejor autoeficacia.
Díaz (2010) incluye 31 jóvenes entre 9 y 17 años con sobrepeso / obesidad y sus padres, utilizando el Programa Cambia, el cual consistió en 12 sesiones consecutivas semanales, 2 horas de grupos en la clínica; e incluía abordar en una de sus fases la actividad física y el uso de técnicas de modificación de conducta, entre otros aspectos. También se reporta un alto nivel de deserción, como en este estudio, que puede llegar hasta el $45 \%$ en lapsos de un año, como en nuestro estudio, tanto para el grupo de intervención como para el grupo control, pero en su caso establece como estrategia la entrevista y medición en el hogar tras el abandono, algo que en nuestro caso no hicimos, y que puede implicar un riesgo de sesgo de los procedimientos de análisis por los datos incompletos.

Carrasco (2009), estudia la relación de la obesidad con los aspectos emocionales en jóvenes entre 14 y 18 ańos, con instrumentos distintos al nuestro, también en dos grupos: control, sin obesidad; y un grupo de estudio con obesidad, y concluye que el grupo de adolescentes obesos presentan dificultades en sus relaciones cercanas, desajustes en el rol social y presentan alto disconfort vital asociado a la presencia de ansiedad y a manifestaciones depresivas.

Las variables de nuestro estudio han sido abordadas por Aedo (2009) y Giménez (2003), con fines de construcción y validación de instrumentos para medir autoeficacia y autoestima, respectivamente. El primer estudio utiliza un muestreo estratificado por grado escolar y sexo, de niños de segundo a quinto grado de primaria, con edades de 7 a 10 años y 11 meses. Una de las conclusiones a las que llegan ambas autoras es semejante s nuestra experiencia: la dificultad de comprensión de los niños de escolaridad básica (de seis a doce ańos) a los instrumentos con escala likert de cuatro o cinco puntos como el de autoeficacia y autoestima. 
Entre las limitaciones en este estudio, se encontró la aplicación de cuestionarios de autoeficacia y autoestima con escala tipo Likert para niños, los cuales tuvieron dificultades de comprensión, que otorga tantos valores a conceptos que para la comunidad en general es más fácil es utilizar opciones dicotómicas (si o no como opciones). Esta limitante ya ha sido reportada en estudios previos en familias latinas por lo cual adaptaron un cuestionario binario con las implicaciones de limitación en el análisis estadístico que implica. Otra limitante fue que la influencia social evaluada en esta investigación que fue solamente determinada por la asistencia de los padres o familiares y determinada por el porcentaje de asistencia. Se reconocen la existencia de cuestionarios de las influencias sociales validados en población México Americana y que requieren de su aplicación en nuestra población (Gessel y cols).
Se propone en investigaciones futuras el evaluar el impacto de las estrategias que fortalecen la autoeficacia en los niños con obesidad y que requieren una validación de cada una de las estrategias por separado y valorar su impacto de forma objetiva y significativa para provocar una mayor actividad física en los niños.

De acuerdo a esta revisión, una gran cantidad de estudios son más bien teóricos, por ejemplo, Guerra (2009), Mejía (2010), Mispireta (2012), Reyna (2012). Los proyectos de investigación- intervención en personas con obesidad se orientan básicamente hacia jóvenes y adultos (según se revisó previamente), y en menor grado, a los niños con obesidad; y los experimentos de campo presentan importantes dificultades para el control riguroso de las variables, como las que se vivieron en este trabajo.

\section{REFERENCIAS}

Aedo, Á., \& Ávila, H. (2009). Nuevo cuestionario para evaluar la autoeficacia hacia la actividad física en nińos. Revista Panameña de Salud Publica, 26(4), 324329.

Alvarado Sánchez, A. M., Guzmán-Benavides, E., \& Ramírez, M. T. G. (2005). Obesidad: ¿Baja Autoestima? Intervención Psicológica en Pacientes con Obesidad. Enseñanza e Investigación en Psicología, 10(2), 417-428.

Ayala, G. X., Elder, J. P., Campbell, N. R., Arredondo, E., Baquero, B., Crespo, N. C., \& Slymen, D. J. (2010). Longitudinal Intervention Effects on Parenting of the Aventuras para Niños Study. American Journal of Preventive Medicine, 38(2), 154-162.

Bandura, A., \& Adams, N. E. (1977). Analysis of Self-Efficacy Theory of Behavioral Change. Cognitive Therapy and Research, 1(4), 287-310.

Bandura, A. (1982). Self- efficacy mechanism in human agency. American Psychologist (2), 122-147.

Beech, B. M., Klesges, R. C., Kumanyika, S. K., Murray, D. M., Klesges, L., McClanahan, B., Slawson, D., Nunnally, C., Rochon, J., McLain-Allen, B., \& Pree-Cary, J. (2003). Child and Parent-Targeted Interventions: The Memphis Gems Pilot Study. Ethnicity \& Disease, 13.

Bersh, S. (2006). La obesidad: aspectos psicológicos y conductuales. Revista Colombiana de Psiquiatría, XXXV(4), 537-546.

Botella-García del Cid, L., Ribas Robert, E., \& Benito Ruíz, J. (2009). Evaluación Psicométrica de la Imagen Corporal: Validación de la Versión Española del Multidimensional Body Self Relations Questionnaire (MBSRQ). Revista Argentina de Clínica Psicológica, 253-264.

Bravo Del Toro, A., Espinoza-Rodríguez, T.,
Mancilla-Arroyo, L. N., \& Tello-Recillas, M. (2011). Rasgos de Personalidad en Pacientes con Obesidad. Enseñanza e Investigación en Psicología, 16(1), 115123.

Calderón, C., Forns, M., \& Varea, V. (2010). Implicación de la ansiedad y la depresión en los trastornos de alimentación de jóvenes con obesidad. Nutrición Hospitalaria, 25(4), 641-647.

Carrasco Ortiz, M. Á., \& del Barrio Gándara, M. V. (2002). Evaluación de la autoeficacia en niños y adolescentes. Psicothema, 14(2), 323-332.

Cole, T., Bellizi, M., Flegal, K., \& Dietz, W. (2000). Establishing a Standard Definition for Child Overweight and Obesity Worldwide International Survey. BMJ, 1240-1243.

Dishman, R., Molt, RW, Saunders, R., \& col, y. (2004). Self-efficacy partially mediate the efect of a school-based physical-activity intervention among adolescent girls. Preventive Medicine, 628-636.

Díaz, R. G., Esparza Romero, J., Moya Camarena, S. Y., Robles Sardín, A. E., \& Valencia, M. E. (2010). Lifestyle Intervention in Primary Care Settings Improves Obesity Parameters among Mexican Youth. American Dietetic Association, 110, 285-289.

Dreyer, M. L., \& Egan, A. M. (2008). Psychosocial functioning and its impact on implementing behavioral interventions for childhood obesity. Progress in Pediatric Cardiology, 25, 159-166.

Gesell, SB; Reynolds, EB; Ip EH y col (2008). Social Influences on Self-Reported Physical Activity in Overweight Latino Children, Clinical Pediatrics,47,797802

Giménez, C., Cortés, M., \& Loaeza, P. (2003). Confiabilidad y validación con niños mexicanos de dos instrumentos que miden la autoestima. Salud Mental, 26(4), 40-46. 
Grembowski, D., Patrick, D., Diehr, P., Durham, M., Beresford, S., Kay, E. \& Hecht, J. (1993). Selfefficacy and behavior among older adults. Journal of Health and Social Behavior, 34, 89-104.

Gutiérrez, J., Rivera, J., Shamah, T., \& Villalpando, M. (2012). Encuesta Nacional de Salud y Nutrición 2012. Resultados Nacionales. Cuernavaca, Morelos: Instituto Nacional de Salud Pública de Mexico.

Guerra Cabrera, C. E., Cabrera-Romero, A. d. C., Santana-Carballosa, I., González-Hermida, A. E., Almaguer-Sabina, P., \& Urra-Coba, T. (2009). Manejo práctico del sobrepeso y la obesidad en la infancia. Revista Electrónica de las Ciencias Médicas en Cienfuegos, 7(1), 61-69.

Kwan, B. M., \& Bryan, A. D. (2009). Affective response to exercise as a component of exercise motivation: Attitudes, norms, self-efficacy, and temporal stability of intentions. Psychology of Sport and Exercise, $1-9$.

Larios, S. E., Ayala, G. X., Arredondo, E. M. Baquero, B., \& Elder, J. P. (2009). Development and validation of scale to measure latino parenting strategies related to children's obesigenic behaviors. The parenting strategies for eating and activity scale (PEAS). Appetite, $52,166-172$.

Liria, R. (2012). Consecuencias de la obesidad en el niño y el adolescente: un problema que requiere atención. Revista Peruana de Medicina Experimental y Salud Pública, 29(3), 357-360.

Martínez Aguilar, M. d. 1. L., Flores-Peña, Y., RizoBaeza, M. d. 1. M., Aguilar-Hernández, R. M., VázquezGalindo, L., \& Gutiérrez-Sánchez, G. (2010). Percepciones de la obesidad de adolescentes obesos estudiantes del $7^{\circ}$ al $9^{\circ}$ grado residentes en Tamaulipas, México. Revista Latino-Americana de Enfermagem, 18(1), 1-7.

Mejía-Botero, F. (2010). Lo educativo en torno a la obesidad y el sobrepeso en México. Revista Latinoamericana, 40(2), 5-8.

Mispireta, M. L. (2012). Determinantes del sobrepeso y la obesidad en niños en edad escolar en perú. Revista Peruana de Medicina Experimental y Salud Pública, 29(3), 361-365.

Molt, R. (2007). Theorical Models for Understanding Physical Activity Behavior Among Children and Adolescents-Social Cognitive Theory and Self Determination Theory. Journal of Teaching in Physical Education, 350-357.

Nowicka, P., Höglund, P., Pietrobelli, A., Lissau, I., \& Flodmark, C.-E. (2008). Family Weight School treatment: 1-year results in obese adolescents. International Journal of Pediatric Obesity, 3, 141-147.

Norton, K., \& Olds, T. (1996). Anthropometrica. Sindey: UNSW Press.

OCDE. Cooperación y Desarrollo Economico (19 de abril de 2013). OECD Organization. Obtenido de Obesity update health 2012: dx.doi.org/10787/88893523956

Olaya, B., Torragona, M. J., de-la-Osa, N., \& Expeleta, L. (2008). Protocolo de evaluación de niños y adolescentes víctimas de la violencia doméstica. Papeles del Psicólogo, 29(1), 123-135.

Olivares, S., Bustos, N., Moreno, X., Lera, L., \& Cortez, S. (2006). Actitudes y prácticas sobre alimentación y actividad física en niños obesos y sus madres en Santiago, chile. Revista chilena de nutrición, 33(2), 170-179.

Quevedo Urías Héctor y Pérez Salvador Blanca Rosa (2008). Estadística para Ingeniería y Ciencias. Grupo Editorial Patria. México. D.F.

Pshycologists, N. A. o. S. (Noviembre). Self-Efficacy: Helping Children Believe They Can Succed. Available: http://www.nasponline.org/publications/cq/pdf/V39 N3_FT_Self-Efficacy.pdf [2013, 16 de abril].

Reyna, L. (2012). Consecuencias de la obesidad en el nińo y el adolescente: Un problema que requiere atención. Rev. Perú Med Exp Salud Publica, 29, 357360.

Rojas-Barahona, C. A., Zegers, B., \& Föster, C. E. (2009). La escala de autoestima de Rosenberg:Validación para Chile en una muestra de jóvenes adultos, adultos y adultos mayores. Revista Médica de Chile(137), 791800.

Saunders, R. P., RR, P., Felton, G., Marsha, D., C, W. M., MA, P., \& Baranowski, T. (1997). Development of Questionnaires to Measure Psychosocial Influences on Children's Physical Activity. Preventive Medicine, 241-247.

Seijas Buschiazzo, D., \& Feuchtmann Sáez, C. (1997). Obesidad: Factores psiquiátricos y psicológicos (26). Boletin de la escuela de medicina. Universidad católica de Chile. Available: http://escuela.med.puc.cl/publ/boletin/obesidad/Obe sidadFactores.html [2013, Abril 2013].

Villaseñor-Bayardo, S. J., Ontiveros-Esqueda, C., \& Cárdenas-Cibrián, K. V. (2006). Salud Mental y Obesidad. Investigación en Salud, 8(2), 86-90. 